\title{
A KOSZUL COMPLEX OVER SKEW POLYNOMIAL RINGS
}

\author{
JOSEP ÀlVAREZ MONTANER, ALBERTO F. BOIX, AND SANTIAGO ZARZUELA \\ Dedicated to Professor Gennady Lyubeznik on the occasion of his 60 th birthday
}

\begin{abstract}
We construct a Koszul complex in the category of left skew polynomial rings associated to a flat endomorphism that provides a finite free resolution of an ideal generated by a Koszul regular sequence.
\end{abstract}

\section{INTRODUCTION}

Let $A$ be a commutative Noetherian ring of characteristic $p>0$ and $F: A \longrightarrow A$ the associated Frobenius map for which $F(a)=a^{p}$ for all $a \in A$. The study of $A$-modules with an action of the Frobenius map has received a lot of attention over the last decades and is at the core of the celebrated theory of tight closure developed by M. Hochster and C. Huneke in [HH90] and the theory of $F$-modules introduced by G. Lyubeznik [Lyu97].

To provide an action of the Frobenius on an $A$-module $M$ is equivalent to give a left $A[\Theta ; F]$ module structure on $M$. Here $A[\Theta ; F]$ stands for the left skew polynomial ring associated to $F$, which is an associative, $\mathbb{N}$-graded, not necessarily commutative ring extension of $A$. More generally, we may also consider the skew polynomial rings $A\left[\Theta ; F^{e}\right]$ associated to the $e$-th iterated Frobenius map and the graded $\operatorname{ring} \mathcal{F}^{M}=\bigoplus_{e>0} \mathcal{F}_{e}^{M}$ introduced by G. Lyubeznik and K. E. Smith in [LS01] that collects all the $A\left[\Theta ; F^{e}\right]$-module structures on $M$ or equivalently, all possible actions of $F^{e}$ on $M$. In the case that $\mathcal{F}^{M}$ is principally generated then it is isomorphic to $A[\Theta ; F]$. We want also to single out here that, under the terminology skew polynomial ring of Frobenius type, Y. Yoshino [Yos94] studied $A[\Theta ; F]$ and left modules over it, and used this study to obtain some new results about tight closure theory; following the same spirit, R. Y. Sharp [Sha09] also studied the ideal theory of $A[\Theta ; F]$. The case when $A$ is a field was studied in detail by F. Enescu [Ene12].

One may also develop a dual notion of right skew polynomial ring associated to $F$ that we denote by $A[\varepsilon ; F]$. A left $A[\varepsilon ; F]$-module structure on $M$ is then equivalent to provide an action of a Cartier operator as considered by M. Blickle and G. Böckle in [BB11]. The corresponding ring collecting all the $A\left[\varepsilon ; F^{e}\right]$-module structures on $M$, that we denote by $\mathcal{C}^{M}=\bigoplus_{e>0} \mathcal{C}_{e}^{M}$, was developed by K. Schwede [Sch11] and M. Blickle [Bli13] (see [BS13a] for a nice survey) and has been a hot topic in recent years because of its role in the theory of test ideals.

Although the theory of skew polynomial rings is classical (see [GW04, Chapter 2] and [MR01, Chapter 1, Sections 2 and 6]), the aim of this work is to go back to its basics and develop new tools that should be potentially useful in the study of modules with a Frobenius or Cartier action. Our approach will be in a slightly more general setting as we will consider a Noetherian commutative ring $A$ (not necessarily of positive characteristic) and a ring homomorphism $\varphi: A \longrightarrow A$. Although

2010 Mathematics Subject Classification. Primary 13A35; Secondary 13B10, 14B25, 16S36.

Key words and phrases. Cartier algebras, Frobenius algebras, skew polynomial rings.

The first author is partially supported by Generalitat de Catalunya 2014SGR-634 project and Spanish Ministerio de Economía y Competitividad MTM2015-69135-P. The second and third authors are supported by Spanish Ministerio de Economía y Competitividad MTM2016-7881-P. The second author is partially supported by the Israel Science Foundation (grant No. 844/14). 
most of the results are mild generalizations of the case of the Frobenius morphism we point out that this general approach has already been fruitful (see [SW07]).

Now, we overview the contents of this paper for the convenience of the reader. In Section 2 we introduce all the basics on left and right skew polynomial rings associated to a morphism $\varphi$ that will be denoted by $A[\Theta ; \varphi]$ and $A[\varepsilon ; \varphi]$. More generally, given an $A$-module $M$, we will consider the corresponding rings $\mathcal{F}^{M, \varphi}$ and $\mathcal{C}^{M, \varphi}$ (see Definition 2.9); both are $\mathbb{N}$-graded, non-commutative rings

$$
\mathcal{F}^{M, \varphi}:=\bigoplus_{e \geq 0} \mathcal{F}_{e}^{M, \varphi}, \quad \mathcal{C}^{M, \varphi}:=\bigoplus_{e \geq 0} \mathcal{C}_{e}^{M, \varphi}
$$

where one collects (see Subsection 2.2) all the possible $\varphi^{e}$-linear maps (respectively, all the possible $\varphi^{-e}$-linear maps). The main result of this Section is Theorem 2.12 which states the following: let $I \subseteq A:=\mathbb{K} \llbracket x_{1}, \ldots, x_{n} \rrbracket$ be an ideal, let $R:=A / I$ and let $E_{R}$ be the injective hull of the residue field of $R$. Then, for any finite morphism $\varphi$, we have an isomorphism between the graded pieces $\mathcal{F}_{e}^{E_{R}, \varphi}$ and $\mathcal{C}_{e}^{R, \varphi}$ induced by Matlis duality.

In Section 3 we present the main result of this work. Here we consider a flat morphism $\varphi$ satisfying some extra condition (which is naturally satisfied in the case of the Frobenius morphism). First we introduce the $\varphi$-Koszul complex which is a Koszul-type complex associated to $x_{1}, \ldots, x_{n} \in A$ in the category of left $A[\Theta ; \varphi]$-modules. In Theorem 3.13 we prove that, whenever $x_{1}, \ldots, x_{n}$ is an $A$-Koszul regular sequence (see Definition 3.12), the $\varphi$-Koszul complex provides a free resolution of $A / I_{n}$ in the category of left $A[\Theta ; \varphi]$-modules, where $I_{n}$ is the ideal generated by $x_{1}, \ldots, x_{n}$. In the case where $A$ is a regular ring of positive characteristic $p>0$ and $\varphi=F^{e}$ is the $e$-th iteration of the Frobenius, we obtain a free resolution, in the category of left $A\left[\Theta ; F^{e}\right]$-modules, of the ideal $I_{n}$ (see Corollary 3.15).

To the best of our knowledge this is one of the first explicit examples of free resolutions of $A$ modules as modules over a skew polynomial ring. We hope that a development of this theory of free resolutions would provide, in the case of the Frobenius morphism, more insight in the study of modules with a Frobenius action.

\section{Preliminaries}

Let $A$ be a commutative Noetherian ring and $\varphi: A \longrightarrow A$ a ring homomorphism. Associated to $\varphi$ we may consider non necessarily commutative algebra extensions of $A$ that are useful in the sense that some non finitely generated $A$-modules become finitely generated when viewed over these extensions. In this section we will collect the basic facts on this theory keeping always an eye on the case of the Frobenius morphism.

First we recall that $\varphi$ allows us to describe a covariant functor, the pushforward or restriction of scalars functor $\varphi_{*}$, from the category of left $A$-modules to the category of left $A$-modules. Namely, given an $A$-module $M, \varphi_{*} M$ is the $(A, A)$-bimodule having the usual $A$-module structure on the right but the left structure is twisted by $\varphi$; that is, if we denote by $\varphi_{*} m(m \in M)$ an arbitrary element of $\varphi_{*} M$ then, for any $a \in A$,

$$
a \cdot \varphi_{*} m:=\varphi_{*}(\varphi(a) m) .
$$

Moreover, given a map $g: M \longrightarrow N$ of left $A$-modules, we can define a map $\varphi_{*} g: \varphi_{*} M \longrightarrow \varphi_{*} N$ of left $A$-modules by setting, for any $m \in M$,

$$
\varphi_{*} g\left(\varphi_{*} m\right):=\varphi_{*} g(m) .
$$


2.1. Left and right skew-polynomial rings. The most basic ring extensions given by a ring homomorphism are the so-called skew-polynomial rings or Ore extensions. This is a classical object of study, and we refer either to [GW04, Chapter 2] or [MR01, Chapter 1, Sections 2 and 6] for further details.

Definition 2.1. Let $A$ be a commutative Noetherian ring and $\varphi: A \longrightarrow A$ a ring homomorphism.

(i) We define the left skew polynomial ring of $A$ determined by $\varphi$ as

$$
A[\Theta ; \varphi]:=\frac{A\langle\Theta\rangle}{\langle\Theta a-\varphi(a) \Theta \mid \quad a \in A\rangle} .
$$

That is, $A[\Theta ; \varphi]$ is a free left $A$-module with basis $\left\{\theta^{i}\right\}_{i \in \mathbb{N}_{0}}$ and $\theta \cdot a=\varphi(a) \theta$ for all $a \in A$.

(ii) We define the right skew polynomial ring of $A$ determined by $\varphi$ as

$$
A[\varepsilon ; \varphi]:=\frac{A\langle\varepsilon\rangle}{\langle a \varepsilon-\varepsilon \varphi(a) \mid a \in A\rangle} .
$$

That is, $A[\varepsilon ; \varphi]$ is a free right $A$-module with basis $\left\{\varepsilon^{i}\right\}_{i \in \mathbb{N}_{0}}$ and $a \cdot \varepsilon=\varepsilon \varphi(a)$ for all $a \in A$.

Remark 2.2. The reader will easily note that $A[\Theta ; \varphi]^{o p} \cong A[\varepsilon ; \varphi]$ and $A[\varepsilon ; \varphi]^{o p} \cong A[\Theta ; \varphi]$, where $(-)^{o p}$ denotes the opposite ring.

Both ring extensions are determined by the following universal property.

Proposition 2.3. Let $B=A[\Theta ; \varphi]$ be the left (resp. $B=A[\varepsilon ; \varphi]$ be the right) skew polynomial ring of $A$ determined by $\varphi$. Suppose that we have a ring $T$, a ring homomorphism $\phi: A \longrightarrow T$ and an element $y \in T$ such that, for each $a \in A$,

$$
y \phi(a)=\phi(\varphi(a)) y \quad(\operatorname{resp} . \phi(a) y=y \phi(\varphi(a))) .
$$

Then, there is a unique ring homomorphism $B \stackrel{\psi}{\longrightarrow} T$ such that $\psi(\Theta)=y($ resp. $\psi(\varepsilon)=y)$ which makes the triangle

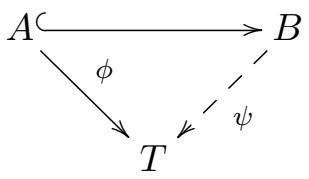

commutative.

This universal property allows us to provide more general examples of skew polynomial rings.

Example 2.4. Let $u \in A$. As $u \Theta$ is an element of $A[\Theta ; \varphi]$ such that, for any $a \in A$,

$$
(u \Theta) a=u \Theta a=u \varphi(a) \Theta=\varphi(a)(u \Theta),
$$

it follows from the universal property for left skew polynomial rings that there is a unique A-algebra homomorphism $A\left[\Theta^{\prime} ; \varphi\right] \longrightarrow A[\Theta ; \varphi]$ sending $\Theta^{\prime}$ to $u \Theta$; we shall denote the image of this map by $A[u \Theta ; \varphi]$. The previous argument shows that $A[u \Theta ; \varphi]$ can be also regarded as a left skew polynomial ring. Analogously, $A[\varepsilon u ; \varphi]$ can be also regarded as right skew polynomial ring since we have, for any $a \in A$,

$$
a(\varepsilon u)=\varepsilon \varphi(a) u=(\varepsilon u) \varphi(a) .
$$

2.1.1. The case of Frobenius homomorphism. We single out the case where $A$ is a ring of positive characteristic $p>0$ and $\varphi=F$ is the Frobenius homomorphism. The specialization of Definition 2.1 to this case is the following:

(i) The Frobenius skew polynomial ring of $A$ is the non-commutative graded $\operatorname{ring} A[\Theta ; F]$ that is, the free left $A$-module with basis $\left\{\Theta^{e}\right\}_{e \in \mathbb{N}_{0}}$ and right multiplication given by

$$
\Theta \cdot a=a^{p} \Theta .
$$


(ii) The Cartier skew polynomial ring of $A$ is the non-commutative graded $\operatorname{ring} A[\varepsilon ; F]$; that is, the free right $A$-module with basis $\left\{\varepsilon^{e}\right\}_{e \in \mathbb{N}_{0}}$ and left multiplication given by

$$
a \cdot \varepsilon=\varepsilon a^{p} \text {. }
$$

It turns out that $A[\Theta ; F]$ is rarely left or right Noetherian, as it is explicitly described by Y. Yoshino in [Yos94, Theorem (1.3)]. He also provided effective descriptions of basic examples of left modules over $A[\Theta ; F]$. Namely, the ring $A$, the localizatons $A_{a}$ at elements $a \in A$ and the local cohomology modules $H_{\mathfrak{m}}^{i}(A)$, where $\mathfrak{m}$ is the maximal ideal of a commutative Noetherian local ring $A$, have an abstract structure as finitely generated left $A[\Theta ; F]$-modules that we collect in the following result. We refer to [Yos94, Lemma (3.2) (b), Lemma (5.1) and Theorem (5.4)] for further details.

Proposition 2.5. Let $A$ be a commutative Noetherian ring of characteristic $p$, let $a \in A$ be any element and let $J(a)$ denote the left ideal of $A[\Theta ; F]$ generated by the infinite set

$$
\left\{a^{p^{e}-1} \Theta^{e}-1 \mid \quad e \in \mathbb{N}\right\} \text {. }
$$

Then, the following statements hold.

(i) A has a natural structure as left $A[\Theta ; F]$-module given by

$$
A \cong A[\Theta ; F] / A[\Theta ; F]\langle\Theta-1\rangle \cong A[\Theta ; F] / J(1) .
$$

Moreover, if $(A, \mathfrak{m}, \mathbb{K})$ is a local ring of characteristic $p$ of dimension $d \geq 1$, then

(ii) If $0 \neq a \in A$, then the localization $A_{a}$ has a natural structure as left $A[\Theta ; F]$-module given by

$$
A_{a} \cong A[\Theta ; F] / J(a)
$$

(iii) The Čech complex of $A$ with respect to a system of parameters $a_{1}, \ldots, a_{d}$ for $A$

$$
0 \longrightarrow A \longrightarrow \bigoplus_{i=1}^{d} A_{a_{i}} \longrightarrow \bigoplus_{1 \leq i<j \leq d} A_{a_{i} a_{j}} \longrightarrow \ldots \longrightarrow \bigoplus_{i=1}^{d} A_{a_{1} \cdots a_{i} \cdots a_{d}} \longrightarrow A_{a_{1} \cdots a_{d}} \longrightarrow 0
$$

is a complex of left $A[\Theta ; F]$-modules which is isomorphic to the following complex:

$$
\begin{aligned}
& 0 \longrightarrow A[\Theta ; F] / J(1) \longrightarrow \bigoplus_{i=1}^{d} A[\Theta ; F] / J\left(a_{i}\right) \longrightarrow \bigoplus_{1 \leq i<j \leq d} A[\Theta ; F] / J\left(a_{i} a_{j}\right) \longrightarrow \ldots \\
& \ldots \longrightarrow \bigoplus_{i=1}^{d} A[\Theta ; F] / J\left(a_{1} \cdots \widehat{a_{i}} \cdots a_{d}\right) \longrightarrow A[\Theta ; F] / J\left(a_{1} \cdots a_{d}\right) \longrightarrow 0 .
\end{aligned}
$$

(iv) Any local cohomology module $H_{\mathfrak{m}}^{i}(A)$ has an abstract structure as a finitely generated left $A[\Theta ; F]$-module; more specifically, one has

$$
H_{\mathfrak{m}}^{d}(A) \cong A[\Theta ; F] /\left(J\left(a_{1} \cdots a_{d}\right)+A[\Theta ; F]\left\langle a_{1}, \ldots, a_{d}\right\rangle\right) .
$$

Remark 2.6. In general, for $A$ being any commutative Noetherian ring of prime characteristic and $I \subseteq A$ being any ideal, it is also true [BS13b, Theorem 5.3.4] that any local cohomology module $H_{I}^{j}(A)$ has an abstract structure as left $A[\Theta ; F]-$ module.

2.2. Skew polynomial rings attached to a ring endomorphism. In this subsection we are going to give a natural generalization of skew polynomial rings associated to a ring homomorphism $\varphi$. Before doing so, we briefly recall how to give a module structure over a skew polynomial ring by means of the so-called $\varphi$ and $\varphi^{-1}$-linear maps.

Definition 2.7. Let $A$ be a commutative Noetherian ring, $\varphi: A \longrightarrow A$ a ring homomorphism and $M$ be an $A$-module. Given $\psi, \phi \in \operatorname{End}_{A}(M)$ :

(i) We say that $\psi$ is $\varphi$-linear provided $\psi(a m)=\varphi(a) \psi(m)$ for any $(a, m) \in A \times M$. 
(ii) We say that $\phi$ is $\varphi^{-1}$-linear provided $\phi(\varphi(a) m)=a \phi(m)$ for any $(a, m) \in A \times M$.

We denote by $\operatorname{End}_{\varphi}(M)$ and $\operatorname{End}_{\varphi^{-1}}(M)$ the $A$-endomorphisms of $M$ which are $\varphi$-linear and $\varphi^{-1}$-linear respectively. These endomorphisms can be interpreted in terms of the pushforward functor since we have the following bijections:

$$
\begin{aligned}
& \operatorname{End}_{\varphi}(M) \longrightarrow \operatorname{Hom}_{A}\left(M, \varphi_{*} M\right), \\
& \operatorname{End}_{\varphi^{-1}}(M) \longrightarrow \operatorname{Hom}_{A}\left(\varphi_{*} M, M\right) \\
& \psi \longmapsto\left[m \longmapsto \varphi_{*}(\psi(m))\right] \\
& \phi \longmapsto\left[\varphi_{*} m \longmapsto \phi(m)\right]
\end{aligned}
$$

An $A[\Theta ; \varphi]$-module is simply an $A$-module $M$ together with a suitable action of $\Theta$ on $M$. Actually, one only needs to consider a $\varphi$-linear map $\psi: M \longrightarrow M$ and define $\Theta \cdot m:=\psi(m)$ for all $m \in M$. Analogously, an $A[\varepsilon ; \varphi]$-module is an $A$-module $M$ together with an action of $\varepsilon$ given by a $\varphi^{-1}$-linear map $\phi: M \longrightarrow M$; we record all these simple remarks into the following:

Proposition 2.8. Let $M$ be an A-module.

(i) There is a bijective correspondence between $\operatorname{End}_{\varphi}(M)$ and the left $A[\Theta ; \varphi]$-module structures which can be attached to $M$.

(ii) There is a bijective correspondence between $\operatorname{End}_{\varphi^{-1}}(M)$ and the left $A[\varepsilon ; \varphi]$-module structures which can be attached to $M$.

More generally, we may consider the $e$-th powers $\varphi^{e}$ of the ring homomorphism $\varphi$ and define the corresponding notion of $\varphi^{e}$ and $\varphi^{-e}$-linear maps. We may collect all these morphisms in a suitable algebra that would provide a generalization of the Frobenius algebra introduced by G. Lyubeznik and K. E. Smith in [LS01, Definition 3.5] and the Cartier algebra considered by K. Schwede [Sch11, pp. 5927-5928] and generalized by M. Blickle [Bli13, Definition 2.2] (see also [BS13a, Definition 9.3.1]).

Definition 2.9. Let $A$ be a commutative Noetherian ring and $\varphi: A \longrightarrow A$ a ring homomorphism, and let $M$ be an $A$-module.

(i) We define the ring of $\varphi$-linear operators on $M$ as the associative, $\mathbb{N}$-graded, not necessarily commutative ring

$$
\mathcal{F}^{M, \varphi}:=\bigoplus_{e \geq 0} \mathcal{F}_{e}^{M, \varphi}
$$

where $\mathcal{F}_{e}^{M, \varphi}:=\operatorname{Hom}_{A}\left(M, \varphi_{*}^{e} M\right)=\operatorname{End}_{\varphi^{e}}(M)$, and the product is given by the rule

$$
\psi_{e^{\prime}} \cdot \psi_{e}:=\varphi_{*}^{e}\left(\psi_{e^{\prime}}\right) \circ \psi_{e} \in \mathcal{F}_{e+e^{\prime}}^{M, \varphi} .
$$

for any given $\psi_{e} \in \mathcal{F}_{e}^{M, \varphi}$ and $\psi_{e^{\prime}} \in \mathcal{F}_{e^{\prime}}^{M, \varphi}$.

(ii) We define the ring of $\varphi^{-1}$-linear operators on $M$ as the associative, $\mathbb{N}$-graded, not necessarily commutative ring

$$
\mathcal{C}^{M, \varphi}:=\bigoplus_{e \geq 0} \mathcal{C}_{e}^{M, \varphi}
$$

where $\mathcal{C}_{e}^{M, \varphi}:=\operatorname{Hom}_{A}\left(\varphi_{*}^{e} M, M\right)=\operatorname{End}_{\varphi^{-e}}(M)$, and the product is given by the rule

$$
\phi_{e^{\prime}} \cdot \phi_{e}:=\phi_{e^{\prime}} \circ \varphi_{*}^{e^{\prime}}\left(\phi_{e}\right) \in \mathcal{C}_{e+e^{\prime}}^{M, \varphi}
$$

for any given $\phi_{e} \in \mathcal{C}_{e}^{M, \varphi}$ and $\phi_{e^{\prime}} \in \mathcal{C}_{e^{\prime}}^{M, \varphi}$.

Actually, the generalization of Cartier algebra given by M. Blickle in [Bli13, Definition 2.2] (see also [BS13a, Definition 9.3.1]) would be interpreted in our context as follows: 
Definition 2.10. An $A$-Cartier algebra with respect to $\varphi$ is an $\mathbb{N}$-graded $A$-algebra

$$
\mathcal{C}^{\varphi}:=\bigoplus_{e \geq 0} \mathcal{C}_{e}^{\varphi}
$$

such that, for any $\left(a, \phi_{e}\right) \in A \times \mathcal{C}_{e}^{\varphi}$, we have that $a \cdot \phi_{e}=\phi_{e} \cdot \varphi^{e}(a)$. The $A$-algebra structure of $\mathcal{C}^{\varphi}$ is given by the structural map from $A$ to $\mathcal{C}_{0}^{\varphi}$. We also assume that this structural map is surjective

Notice that this notion of Cartier algebra does not depend on a specific module; moreover, we also observe that $\mathcal{C}^{M, \varphi}$ is generally not an $A$-Cartier algebra with respect to $\varphi$, since $\mathcal{C}_{0}^{M, \varphi}=\operatorname{End}_{A}(M)$ and therefore the natural map $A \longrightarrow \operatorname{End}_{A}(M)$ that sends $a \in A$ to the multiplication by $a$ map on $M$ is, in general, not surjective. Nevertheless, if $M=A / I$ (where $I$ is any ideal of $A$ ) then $\operatorname{End}_{A}(M)=A / I$ and therefore it follows that $\mathcal{C}^{A / I, \varphi}$ is an $A$-Cartier algebra.

Whenever the ring of $\varphi$-linear (resp. $\varphi^{-1}$-linear) operators is principally generated it is isomorphic to a left (resp. right) skew polynomial ring. This is the case of the ring of $\varphi$-linear operators of the ring $A$. This was already observed by G. Lyubeznik and K. E. Smith [LS01, Example 3.6] when $A$ is of prime characteristic and $\varphi=F$ is the Frobenius map.

Example 2.11. We have that $\mathcal{F}^{A, \varphi} \cong A[\Theta ; \varphi]$. Indeed, fix $e \in \mathbb{N}$ and let $\psi_{e} \in \mathcal{F}_{e}^{A, \varphi}$. We point out that, for any $a \in A$,

$$
\psi_{e}(a)=\psi_{e}(a \cdot 1)=\varphi^{e}(a) \psi_{e}(1)=\psi_{e}(1) \varphi^{e}(a)
$$

In this way, set

$$
\begin{gathered}
\mathcal{F}_{e}^{A, \varphi} \stackrel{b_{e}}{\longrightarrow} A \Theta^{e} \\
\psi_{e} \longmapsto \psi_{e}(1) \Theta^{e} .
\end{gathered}
$$

The previous straightforward calculation shows the injectivity of this map. In fact, it is a bijective map with inverse

$$
\begin{gathered}
A \Theta^{e} \longrightarrow \mathcal{F}_{e}^{A, \varphi} \\
a \Theta^{e} \longmapsto a \varphi^{e} .
\end{gathered}
$$

In this way, setting $\mathcal{F}^{A, \varphi} \stackrel{b}{\longrightarrow} A[\Theta ; \varphi]$ as the unique map of rings given in degree $e$ by $b_{e}$ it follows that $b$ is an isomorphism of graded algebras.

2.2.1. Duality between Cartier algebras and Frobenius algebras. Let $A=\mathbb{K} \llbracket x_{1}, \ldots, x_{d} \rrbracket$ be a formal power series ring with $d$ indeterminates over a field $\mathbb{K}, I \subseteq A$ an ideal and $R:=A / I$. Let $\varphi: A \longrightarrow A$ be a ring homomorphism such that $\varphi_{*}^{e} A$ is finitely generated as $A$-module. The aim of this subsection is to establish an explicit correspondence, at the level of graded pieces, between $\mathcal{C}^{R, \varphi}$ and $\mathcal{F}^{E_{R}, \varphi}$ given by Matlis duality. This would extend the correspondence given in the case of the Frobenius map (cf. [BB11, Proposition 5.2] and [SY11, Theorem 1.20 and Corollary 1.21]).

Theorem 2.12. Let $\varphi: A \longrightarrow A$ be a ring homomorphism such that $\varphi_{*}^{e} A$ is finitely generated as $A$-module. Then we have that

$$
\operatorname{Hom}_{A}\left(\varphi_{*}^{e} R, R\right)^{\vee} \cong \operatorname{Hom}_{A}\left(E_{R}, \varphi_{*}^{e} E_{R}\right) \quad \text { and } \operatorname{Hom}_{A}\left(E_{R}, \varphi_{*}^{e} E_{R}\right)^{\vee} \cong \operatorname{Hom}_{A}\left(\varphi_{*}^{e} R, R\right) .
$$

Before proving this theorem we have to show a previous statement which we shall need during its proof; albeit the below result was obtained by F. Enescu and M. Hochster in [EH08, Discussion (3.4)] (see also [Yos94, Lemma (3.6)]), we review here their proof for the convenience of the reader.

Lemma 2.13. Let $(A, \mathfrak{m}, \mathbb{K}) \longrightarrow(B, \mathfrak{n}, \mathbb{L})$ be a local homomorphism of local rings, and suppose that $\mathfrak{m} B$ is $\mathfrak{n}$-primary and that $\mathbb{L}$ is finite algebraic over $\mathbb{K}$ (both these conditions hold if $B$ is modulefinite over $A)$. Let $E:=E_{A}(\mathbb{K})$ and $E_{B}(\mathbb{L})$ denote choices of injective hulls for $\mathbb{K}$ over $A$ and for $\mathbb{L}$ 
over $B$, respectively. Then, the functor $\operatorname{Hom}_{A}(-, E)$, on $B$-modules, is isomorphic with the functor $\operatorname{Hom}_{B}\left(-, E_{B}(\mathbb{L})\right)$.

Proof. First of all, we underline that $\operatorname{Hom}_{A}(-, E)$, on $B$-modules, can be identified via adjunction with

$$
\operatorname{Hom}_{A}\left((-) \otimes_{A} B, E\right) \cong \operatorname{Hom}_{B}\left(-, \operatorname{Hom}_{A}(B, E)\right)
$$

and therefore $\operatorname{Hom}_{A}(B, E)$ is injective as $B$-module. Moreover, as $\mathfrak{m} B$ is $\mathfrak{n}$-primary any element of $\operatorname{Hom}_{A}(B, E)$ is killed by a power of $\mathfrak{n}$ and therefore

$$
\operatorname{Hom}_{A}(B, E) \cong E_{B}(\mathbb{L})^{\oplus l} .
$$

In this way, it only remains to check that $l=1$. Indeed, we note that

$$
\operatorname{Hom}_{A}(\mathbb{L}, E) \cong \operatorname{Hom}_{A}(\mathbb{L}, \mathbb{K}) .
$$

However, as $A$-module, $\operatorname{Hom}_{A}(\mathbb{L}, \mathbb{K})$ is abstractly isomorphic to $\mathbb{L}$ (here we are using the assumption that $\mathbb{L}$ is finite algebraic over $\mathbb{K}$ ). Thus, all these foregoing facts imply that

$$
E_{B}(\mathbb{L}) \cong \operatorname{Hom}_{A}(B, E),
$$

hence $\operatorname{Hom}_{A}\left((-) \otimes_{A} B, E\right) \cong \operatorname{Hom}_{B}\left(-, E_{B}(\mathbb{L})\right)$ and we get the desired conclusion.

Proof of Theorem 2.12. First of all, we underline that

$$
\operatorname{Hom}_{A}\left(\varphi_{*}^{e} R, R\right)^{\vee} \cong \operatorname{Hom}_{A}\left(E_{R}, \varphi_{*}^{e}(R)^{\vee}\right) .
$$

Now, let $E_{*}$ be the injective hull of the residue field of $\varphi_{*}^{e} R$ and recall that $E=E_{A}(\mathbb{K})$. In this way, from Lemma 2.13 we deduce that $\operatorname{Hom}_{A}(-, E) \cong \operatorname{Hom}_{\varphi_{*}^{e} A}\left(-, E_{*}\right)$ as functors of $\varphi_{*}^{e} A$-modules. Therefore, combining all these facts joint with the exactness of $\varphi_{*}^{e}$ it follows that

$$
\varphi_{*}^{e}(R)^{\vee} \cong \operatorname{Hom}_{A}\left(\varphi_{*}^{e} R, E\right) \cong \operatorname{Hom}_{\varphi_{*}^{e} A}\left(\varphi_{*}^{e} R, E_{*}\right) \cong \varphi_{*}^{e} \operatorname{Hom}_{A}(R, E) \cong \varphi_{*}^{e} E_{R} .
$$

Thus, taking into account this last chain of isomorphisms one obtains the first desired conclusion.

On the other hand, using once more Lemma 2.13 it turns out that

$$
\varphi_{*}^{e}\left(E_{R}\right)^{\vee} \cong \operatorname{Hom}_{A}\left(\varphi_{*}^{e} E_{R}, E\right) \cong \operatorname{Hom}_{\varphi_{*}^{e} A}\left(\varphi_{*}^{e} E_{R}, \varphi_{*}^{e} E\right) \cong \varphi_{*}^{e}\left(E_{R}^{\vee}\right) \cong \varphi_{*}^{e} R .
$$

Thus, bearing in mind this last chain of isomorphisms it follows that

$$
\operatorname{Hom}_{A}\left(E_{R}, \varphi_{*}^{e} E_{R}\right)^{\vee} \cong \operatorname{Hom}_{A}\left(\varphi_{*}^{e}\left(E_{R}\right)^{\vee}, E_{R}^{\vee}\right) \cong \operatorname{Hom}_{A}\left(\varphi_{*}^{e} R, R\right),
$$

just what we finally wanted to show.

2.2.2. The case of Frobenius homomorphism. Once again we single out the case where $A$ is a ring of positive characteristic $p>0$ and $\varphi=F$ is the Frobenius homomorphism. In this case we adopt the terminology of $p^{e}$ and $p^{-e}$-linear maps or, following [And00], Frobenius and Cartier linear maps.

Definition 2.14. Let $M$ be an $A$-module and $\psi, \phi \in \operatorname{End}_{A}(M)$.

(i) We say that $\psi$ is $p^{e}$-linear provided $\psi(a m)=a^{p^{e}} \psi(m)$ for any $(a, m) \in A \times M$. Equivalently, $\psi \in \operatorname{Hom}_{A}\left(M, F_{*}^{e} M\right)$.

(ii) We say that $\phi$ is $p^{-e}$-linear provided $\phi\left(a^{p^{e}} m\right)=a \phi(m)$ for any $(a, m) \in A \times M$. Equivalently, $\phi \in \operatorname{Hom}_{A}\left(F_{*}^{e} M, M\right)$.

The corresponding rings of $p^{e}$ and $p^{-e}$-linear maps are defined as follows:

Definition 2.15. Let $A$ be a commutative Noetherian ring of prime characteristic $p$ and let $M$ be an $A$-module. 
(i) The Frobenius algebra attached to $M$ is the associative, $\mathbb{N}$-graded, not necessarily commutative ring

$$
\mathcal{F}^{M}:=\bigoplus_{e \geq 0} \operatorname{Hom}_{A}\left(M, F_{*}^{e} M\right) .
$$

(ii) The Cartier algebra attached to $M$ is the associative, $\mathbb{N}$-graded, not necessarily commutative ring

$$
\mathcal{C}^{M}:=\bigoplus_{e \geq 0} \operatorname{Hom}_{A}\left(F_{*}^{e} M, M\right)
$$

In this work we are mainly interested in the case where the Frobenius (resp. Cartier) algebra of a module is principally generated and thus isomorphic to the left (resp. right) skew polynomial ring. G. Lyubeznik and K. E. Smith already carried out such an example in [LS01, Example 3.7].

Example 2.16. Let $(A, \mathfrak{m}, \mathbb{K})$ be a local ring of characteristic $p$. Then

$$
\mathcal{F}^{H_{\mathfrak{m}}^{\operatorname{dim}(A)}(A) \cong S[\Theta ; F]}
$$

where $S$ denotes the $S_{2}$-ification of the completion $\widehat{A}$.

Another source of examples is given by the following result.

Proposition 2.17. Let $(A, \mathfrak{m}, \mathbb{K})$ be a complete $F$-finite local ring of characteristic $p$ and $E_{A}$ stands for a choice of injective hull of $\mathbb{K}$ over $A$. Then, the following statements hold.

(i) If $A$ is quasi Gorenstein then $\mathcal{F}^{E_{A}}$ is principal.

(ii) If $A$ is a $\mathbb{Q}$-Gorenstein normal domain then $\mathcal{F}^{E_{A}}$ is a finitely generated A-algebra if and only if $p$ is relatively prime with the index of $A$.

(iii) If $A$ is a $\mathbb{Q}$-Gorenstein normal domain then $\mathcal{F}^{E_{A}}$ is principal if and only if the index of $A$ divides $p-1$.

Sketch of proof. If $A$ is quasi Gorenstein then $E_{A} \cong H_{\mathfrak{m}}^{\operatorname{dim}(A)}(A)$. But we have seen in Example 2.16 that, under our assumptions, $\mathcal{F}^{H_{\mathfrak{m}}^{\operatorname{dim}(A)}}(A) \cong A[\Theta ; F]$; indeed, $A$ is complete and any quasi Gorenstein ring is, in particular, $S_{2}$. On the other hand, part (ii) follows essentially in the following way; let $m$ be the index of $A$. If $m$ is relatively prime to $p$, then $p$ is a unit modulo $m$, and therefore there is an integer $e_{0} \geq 1 \mathrm{such}$ that $p^{e_{0}} \equiv 1(\bmod m)$. What is proved in [KSSZ14, Proposition 4.1] is that $\mathcal{F}^{E_{A}}$ is generated over $A$ by homogeneous elements of degree at most $e_{0}$. On the other hand, if $p$ divides $m$ then it is shown in [EY16, Theorem 4.5], building upon the isomorphism between $\mathcal{F}^{E_{A}}$ and the so-called $T$-construction of the anticanonical cover of $A$ (see e.g. [KSSZ14, Theorem 3.3 (2)] or [EY16, Theorem 4.1]), that $\mathcal{F}^{E_{A}}$ is not a finitely generated $A$-algebra. Finally, part (iii) again follows essentially from the isomorphism between $\mathcal{F}^{E_{A}}$ and the so-called $T$-construction of the anticanonical cover of $A$ [EY16, Proposition 4.2].

We point out that an explicit description of $\mathcal{F}^{E_{A}}$ can be obtained using the following result due to R. Fedder (cf. [Fed83, pp. 465]).

Theorem 2.18. Let $A=\mathbb{K} \llbracket x_{1}, \ldots, x_{d} \rrbracket$ be a formal power series ring over a field $\mathbb{K}$ of prime characteristic $p$. Let $I$ be an arbitrary ideal of $A, R:=A / I, E$ denotes a choice of injective hull of $\mathbb{K}$ over $A, \Theta$ is the standard Frobenius action on $E$ and $E_{R}:=\left(0:_{E} I\right)$. Then, one has that

$$
\mathcal{F}^{E_{R}} \cong \bigoplus_{e \geq 0}\left\{\left(I^{\left[p^{e}\right]}:_{A} I\right) / I^{\left[p^{e}\right]}\right\} \Theta^{e} .
$$

In [ÀMBZ12] we used this result to study Frobenius algebras associated to Stanley-Reisner rings. It turns out that, whenever they are principally generated, they are isomorphic to $A[u \Theta ; F]$ with $u=x_{1}^{p-1} \cdots x_{d}^{p-1}$. 


\section{The $\varphi$-Koszul Chain COMPlex}

Let $S=\mathbb{K}\left[x_{1}, \ldots, x_{n}\right]$ be a polynomial ring with coefficients on a commutative ring $\mathbb{K}$, and let $\varphi: S \longrightarrow S$ be a flat map of $\mathbb{K}$-algebras satisfying the extra condition that for any $1 \leq i \leq n$, $\varphi\left(x_{i}\right) \in\left\langle x_{i}\right\rangle$. Thus, there are non-zero elements $s_{1}, \ldots, s_{n}$ of $S$ such that $\varphi\left(x_{i}\right)=s_{i} x_{i}$, for each $1 \leq i \leq n$.

Remark 3.1. If $\mathbb{K}$ is a field of positive characteristic $p>0$ and $\varphi=F^{e}$ is the iterated Frobenius morphism, then this extra condition is naturally satisfied. Indeed, $F^{e}\left(x_{i}\right)=x_{i}^{p^{e}}$ so $s_{i}=x_{i}^{p^{e}-1}$. More generally [SW07, Example 2.2], if $\mathbb{K}$ is any field and $t \geq 1$ is an integer, then the $\mathbb{K}$-linear map $\varphi$ on $S$ sending each $x_{i}$ to $x_{i}^{t}$ also satisfies this condition; indeed, in this case, $s_{i}=x_{i}^{t-1}$.

Our aim is to construct a Koszul complex in the category of $S[\Theta ; \varphi]$-modules that we will denote as $\varphi$-Koszul complex. To begin with, let us fix some notations. Let

$$
K_{\bullet}:=K_{\bullet}\left(x_{1}, \ldots, x_{n}\right):=0 \longrightarrow K_{n} \stackrel{d_{n}}{\longrightarrow} K_{n-1} \longrightarrow \ldots \longrightarrow K_{2} \stackrel{d_{2}}{\longrightarrow} K_{1} \stackrel{d_{1}}{\longrightarrow} K_{0} \longrightarrow 0
$$

be the Koszul chain complex of $S$ with respect to $x_{1}, \ldots, x_{n}$ (regarded as a chain complex in the category of left $S$-modules) and suppose that each differential $d_{l}$ is represented by right multiplication by a matrix $M_{l}$. Moreover, for each $l \geq 0 M_{l}^{[\varphi]}$ denotes the matrix obtained by applying to each entry of $M_{l}$ the map $\varphi$. In particular, for each entry $m$, the sign of $m$ is equal to the sign of $\varphi(m)$.

Definition 3.2. We define the $\varphi$-Koszul chain complex with respect to $x_{1}, \ldots, x_{n}$ as the chain complex

$$
\text { FK. }\left(x_{1}, \ldots, x_{n}\right):=0 \longrightarrow \mathrm{FK}_{n+1} \stackrel{\partial_{n+1}}{\longrightarrow} \mathrm{FK}_{n} \stackrel{\partial_{n}}{\longrightarrow} \ldots \stackrel{\partial_{1}}{\longrightarrow} \mathrm{FK}_{0} \longrightarrow 0 .
$$

Here, for each $0 \leq l \leq n+1$,

$$
\mathrm{FK}_{l}:=\bigoplus_{1 \leq i_{1}<\ldots<i_{l} \leq n} S[\Theta ; \varphi]\left(\mathbf{e}_{i_{1}} \wedge \ldots \wedge \mathbf{e}_{i_{l}}\right) \oplus \bigoplus_{1 \leq j_{1}<\ldots<j_{l-1} \leq n} S[\Theta ; \varphi]\left(\mathbf{e}_{j_{1}} \wedge \ldots \wedge \mathbf{e}_{j_{l-1}} \wedge u\right),
$$

where $\mathbf{e}_{1}, \ldots, \mathbf{e}_{n}$ correspond respectively to $x_{1}, \ldots, x_{n}$ and $u$ corresponds to $\Theta-1$. Here, we are adopting the convention that $\mathrm{FK}_{0}:=S[\Theta ; \varphi]$.

Moreover, one defines $\mathrm{FK}_{l} \stackrel{\partial_{l}}{\longrightarrow} \mathrm{FK}_{l-1}$ as the unique homomorphism of left $S[\Theta ; \varphi]$-modules which, on basic elements, acts in the following manner:

. Given $1 \leq i_{1}<\ldots<i_{l} \leq n$, set

$$
\partial_{l}\left(\mathbf{e}_{i_{1}} \wedge \ldots \wedge \mathbf{e}_{i_{l}}\right):=\sum_{r=1}^{l}(-1)^{r-1} x_{i_{r}}\left(\mathbf{e}_{i_{1}} \wedge \ldots \wedge \mathbf{e}_{i_{r-1}} \wedge \mathbf{e}_{i_{r+1}} \wedge \ldots \wedge \mathbf{e}_{i_{l}}\right) .
$$

- Given $1 \leq j_{1}<\ldots<j_{l-1} \leq n$, set

$$
\begin{aligned}
& \partial_{l}\left(\mathbf{e}_{j_{1}} \wedge \ldots \wedge \mathbf{e}_{j_{l-1}} \wedge u\right):=(-1)^{l-1}\left(\Theta-\left(s_{j_{1}} \ldots s_{j_{l-1}}\right)\right)\left(\mathbf{e}_{j_{1}} \wedge \ldots \wedge \mathbf{e}_{j_{l-1}}\right) \\
& +\sum_{r=1}^{l-1}(-1)^{r-1} \varphi\left(x_{j_{r}}\right)\left(\mathbf{e}_{j_{1}} \wedge \ldots \wedge \mathbf{e}_{j_{r-1}} \wedge \mathbf{e}_{j_{r+1}} \wedge \ldots \wedge \mathbf{e}_{j_{l-1}} \wedge u\right) .
\end{aligned}
$$

Before going on, we make the following useful

Discussion 3.3. Given a free, finitely generated left $S$-module $M$ (whence $M$ is abstractly isomorphic to $S^{\oplus r}$ for some $r \in \mathbb{N}$ ), we denote by $S[\Theta ; \varphi] \otimes_{S} S^{\oplus r} \stackrel{\lambda_{M}}{\longrightarrow} S[\Theta ; \varphi]^{\oplus r}$ the natural isomorphism 
of left $S[\Theta ; \varphi]$-modules given by the assignment $s \otimes m \longmapsto s m$; the reader will easily note that, for each $0 \leq l \leq n$, we have the following commutative diagram:

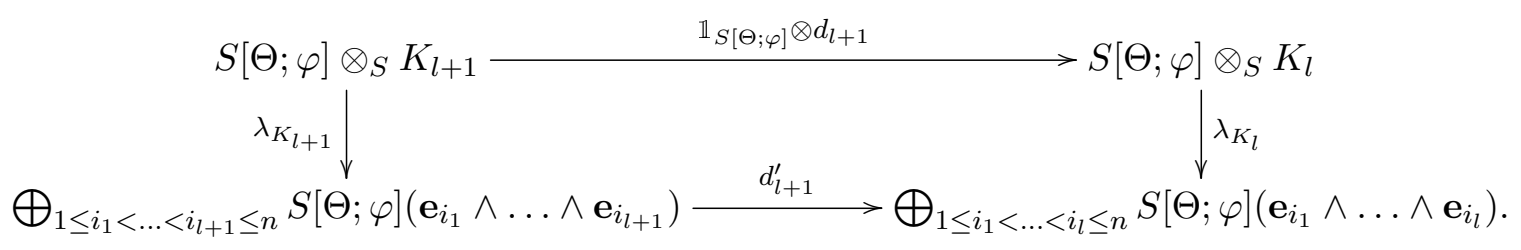

Here, $d_{l+1}^{\prime}$ denotes the map $\partial_{l+1}$ restricted to the direct summand

$$
\bigoplus_{1 \leq i_{1}<\ldots<i_{l+1} \leq n} S[\Theta ; \varphi]\left(\mathbf{e}_{i_{1}} \wedge \ldots \wedge \mathbf{e}_{i_{l+1}}\right) .
$$

As we shall see quickly, this fact turns out to be very useful in what follows.

The first thing we have to check out is that $\mathrm{FK}_{\bullet}\left(x_{1}, \ldots, x_{n}\right)$ defines a chain complex in the category of left $S[\Theta ; \varphi]$-modules. This fact follows from the next:

Proposition 3.4. For any $0 \leq l \leq n$, one has that $\partial_{l} \partial_{l+1}=0$.

Proof. Regarding the very definition of the $\partial$ 's, we only have to distinguish two cases.

. Given $1 \leq i_{1}<\ldots<i_{l+1} \leq n$ one has, keeping in mind Discussion 3.3, that

$$
\begin{aligned}
& \partial_{l}\left(\partial_{l+1}\left(\mathbf{e}_{i_{1}} \wedge \ldots \wedge \mathbf{e}_{i_{l+1}}\right)\right)=d_{l}^{\prime}\left(d_{l+1}^{\prime}\left(\mathbf{e}_{i_{1}} \wedge \ldots \wedge \mathbf{e}_{i_{l+1}}\right)\right) \\
& =\left(\lambda_{K_{l-1}} \circ\left(\mathbb{1}_{S[\Theta ; \varphi]} \otimes d_{l-1}\right) \circ \lambda_{K_{l}}^{-1}\right) \circ\left(\lambda_{K_{l}} \circ\left(\mathbb{1}_{S[\Theta ; \varphi]} \otimes d_{l}\right) \circ \lambda_{K_{l+1}}^{-1}\right)\left(\mathbf{e}_{i_{1}} \wedge \ldots \wedge \mathbf{e}_{i_{l+1}}\right) \\
& =\left(\lambda_{K_{l-1}} \circ\left(\mathbb{1}_{S[\Theta ; \varphi]} \otimes\left(d_{l} \circ d_{l+1}\right)\right) \circ \lambda_{K_{l+1}}^{-1}\right)\left(\mathbf{e}_{i_{1}} \wedge \ldots \wedge \mathbf{e}_{i_{l+1}}\right) \\
& =\left(\lambda_{K_{l-1}} \circ\left(\mathbb{1}_{S[\Theta ; \varphi]} \otimes 0\right) \circ \lambda_{K_{l+1}}^{-1}\right)\left(\mathbf{e}_{i_{1}} \wedge \ldots \wedge \mathbf{e}_{i_{l+1}}\right)=0 ;
\end{aligned}
$$

indeed, notice that $d_{l} d_{l+1}=0$ because they are the usual chain differentials in the Koszul chain complex of $S$ with respect to $x_{1}, \ldots, x_{n}$.

- Given $1 \leq j_{1}<\ldots<j_{l} \leq n$, and setting, for each $1 \leq t, s \leq l$,

$$
\begin{aligned}
& \mathbf{e}_{J_{t}}:=\mathbf{e}_{j_{1}} \wedge \ldots \wedge \mathbf{e}_{j_{t-1}} \wedge \mathbf{e}_{j_{t+1}} \wedge \ldots \wedge \mathbf{e}_{j_{l}}, \\
& \mathbf{e}_{J_{t s}}:=\mathbf{e}_{j_{1}} \wedge \ldots \wedge \mathbf{e}_{j_{t-1}} \wedge \mathbf{e}_{j_{t+1}} \wedge \ldots \wedge \mathbf{e}_{j_{s-1}} \wedge \mathbf{e}_{j_{s+1}} \wedge \ldots \wedge \mathbf{e}_{j_{l}},
\end{aligned}
$$

one has that

$$
\begin{aligned}
& \partial_{l}\left(\partial_{l+1}\left(\mathbf{e}_{j_{1}} \wedge \ldots \wedge \mathbf{e}_{j_{l}} \wedge u\right)\right)=\partial_{l}\left((-1)^{l}\left(\Theta-\left(s_{j_{1}} \cdots s_{j_{l}}\right)\right)\left(\mathbf{e}_{j_{1}} \wedge \ldots \wedge \mathbf{e}_{j_{l}}\right)+\right. \\
& \left.\sum_{r=1}^{l}(-1)^{r-1} \varphi\left(x_{j_{r}}\right)\left(\mathbf{e}_{J_{r}} \wedge u\right)\right)=\sum_{r=1}^{l}(-1)^{r+l-1}\left(\varphi\left(x_{j_{r}}\right) \Theta-\left(s_{j_{1}} \cdots s_{j_{l}}\right) x_{j_{r}}\right) \mathbf{e}_{J_{r}}+ \\
& \sum_{r=1}^{l} \sum_{k=1}^{r-1}(-1)^{r+k-2} \varphi\left(x_{j_{k}} x_{j_{r}}\right)\left(\mathbf{e}_{J_{k r}} \wedge u\right)+\sum_{r=1}^{l} \sum_{k=r+1}^{l}(-1)^{r+k-3} \varphi\left(x_{j_{k}} x_{j_{r}}\right)\left(\mathbf{e}_{J_{r k}} \wedge u\right)+ \\
& \sum_{r=1}^{l}(-1)^{l+r-2}\left(\varphi\left(x_{j_{r}}\right) \Theta-\left(s_{j_{1}} \cdots s_{j_{r-1}} s_{j_{r+1}} s_{j_{r+2}} \cdots s_{j_{l}}\right) \varphi\left(x_{j_{r}}\right)\right) \mathbf{e}_{J_{r}} .
\end{aligned}
$$

Starting from the top, the first summand cancels out the fourth summand just because $\varphi\left(x_{j_{r}}\right)=$ $s_{j_{r}} x_{j_{r}}$; on the other hand, the second summand cancels out the third summand because $\mathbf{e}_{J_{k r}}=$ $-\mathbf{e}_{J_{r k}}$. Therefore, the whole expression vanishes, just what we finally wanted to check. 
Remark 3.5. The differentials of the $\varphi$-Koszul complex

$$
\mathrm{FK}_{\bullet}\left(x_{1}, \ldots, x_{n}\right):=0 \longrightarrow \mathrm{FK}_{n+1} \stackrel{\partial_{n+1}}{\longrightarrow} \mathrm{FK}_{n} \stackrel{\partial_{n}}{\longrightarrow} \ldots \stackrel{\partial_{1}}{\longrightarrow} \mathrm{FK}_{0} \longrightarrow 0 .
$$

are described as follows:

(i) $\partial_{n+1}$ is represented by right multiplication by the matrix $\left((-1)^{n}\left(\Theta-\left(s_{1} \cdots s_{n}\right)\right) \quad M_{n}^{[\varphi]}\right)$.

(ii) For each $1 \leq l \leq n-1, \partial_{l+1}$ is represented by right multiplication by the matrix

$$
\left(\begin{array}{c|c}
M_{l+1} & \mathbf{0} \\
\hline(-1)^{l} D_{l} & M_{l}^{[\varphi]}
\end{array}\right)
$$

where $D_{l}$ is a diagonal matrix with non-zero entries $\Theta-\left(s_{i_{1}} \cdots s_{i_{l}}\right), 1 \leq i_{1}<\ldots<i_{l} \leq n$. (iii) $\partial_{1}$ is represented by right multiplication by the matrix $\left(\begin{array}{llll}x_{1} & \ldots & x_{n} & \Theta-1\end{array}\right)^{T}$.

For example, when $n=2$, FK. $\left(x_{1}, x_{2}\right)$ boils down to the chain complex

$$
0 \longrightarrow S[\Theta ; \varphi] \stackrel{\partial_{3}}{\longrightarrow} S[\Theta ; \varphi]^{\oplus 3} \stackrel{\partial_{2}}{\longrightarrow} S[\Theta ; \varphi]^{\oplus 3} \stackrel{\partial_{1}}{\longrightarrow} S[\Theta ; \varphi] \longrightarrow 0,
$$

where $\partial_{3}, \partial_{2}$ and $\partial_{1}$ are given by right multiplication by matrices

$$
\left(\begin{array}{lll}
\Theta-\left(s_{1} s_{2}\right) & -\varphi\left(x_{2}\right) & \varphi\left(x_{1}\right)
\end{array}\right) \quad\left(\begin{array}{cc|c}
-x_{2} & x_{1} & 0 \\
\hline s_{1}-\Theta & 0 & \varphi\left(x_{1}\right) \\
0 & s_{2}-\Theta & \varphi\left(x_{2}\right)
\end{array}\right) \quad\left(\begin{array}{c}
x_{1} \\
x_{2} \\
\Theta-1
\end{array}\right)
$$

Before showing our main result, we want to establish a certain technical fact, which is interesting in its own right; namely:

Proposition 3.6. $S[\Theta ; \varphi]$ is a flat right $S$-module.

Proof. By the very definition of left skew polynomial rings,

$$
S[\Theta ; \varphi]=\bigoplus_{e \geq 0} S \Theta^{e} .
$$

Since a direct sum of right $S$-modules is flat if and only if so all its direct summands are [Rot09, Proposition 3.46 (ii)], it is enough to check that, for any $e \geq 0, S \Theta^{e}$ is a flat right $S$-module.

Fix $e \geq 0$. Firstly, albeit the notation $S \Theta^{e}$ might suggest that it is just a left $S$-module, this is not the case because $S \Theta^{e}$ can be identified with $\Theta^{e} \varphi_{*}^{e} S$; from this point of view, it is clear that $S \Theta^{e}$ may be also regarded as a right $S$-module. Therefore, keeping in mind the previous identification one has that the map $\Theta^{e} \varphi_{*}^{e} S \longrightarrow \varphi_{*}^{e} S$ given by the assignment $\Theta^{e} \varphi_{*}^{e} s \longmapsto \varphi_{*}^{e} s$ defines an abstract isomorphism of right $S$-modules, whence $S \Theta^{e}$ is (abstractly) isomorphic to $\varphi_{*}^{e} S$ in the category of right $S$-modules and then the result follows from the fact that $\varphi_{*}^{e} S$ is a flat right $S$-module because of the flatness of $\varphi$; the proof is therefore completed.

Remark 3.7. When $S$ is a commutative Noetherian regular local ring of prime characteristic $p$ and $F$ is the Frobenius map on $S$, the fact that $S[\Theta ; F]$ is a flat right $S$-module was already observed by Y. Yoshino [Yos94, Proof of Example (9.2)].

Next result provides some useful properties of FK. $\left(x_{1}, \ldots, x_{n}\right)$.

Proposition 3.8. $H_{0}\left(\mathrm{FK}_{\bullet}\left(x_{1}, \ldots, x_{n}\right)\right) \cong S / I_{n}$ as left $S[\Theta ; \varphi]$-modules, where $I_{n}=\left\langle x_{1}, \ldots, x_{n}\right\rangle$; moreover, $H_{n+1}\left(\mathrm{FK}_{\bullet}\left(x_{1}, \ldots, x_{n}\right)\right)=0$.

Proof. First we notice that, as in the case of the Frobenius morphism studied by Y. Yoshino (see Proposition 2.5), we have $\frac{S[\Theta ; \varphi]}{S[\Theta ; \varphi](\Theta-1)} \cong S$. Then, using Remark 3.5 it follows that

$$
H_{0}\left(\mathrm{FK}_{\bullet}\left(x_{1}, \ldots, x_{n}\right)\right)=\frac{S[\Theta ; \varphi]}{\operatorname{Im}\left(\partial_{1}\right)} \cong \frac{S[\Theta ; \varphi]}{S[\Theta ; \varphi] I_{n}+S[\Theta ; \varphi](\Theta-1)} \cong S / I_{n} .
$$


Now, consider the composition

$$
S[\Theta ; \varphi] \stackrel{\partial_{n+1}}{\longrightarrow} S[\Theta ; \varphi] \oplus S[\Theta ; \varphi]^{\oplus n} \stackrel{\pi}{\longrightarrow} S[\Theta ; \varphi]^{\oplus n},
$$

where $\pi$ denotes the corresponding projection. In this way, we have that $\pi \partial_{n+1}$ turns out to be, up to isomorphisms, $\mathbb{1}_{S[\Theta ; \varphi]} \otimes d_{n}^{[\varphi]}$ (indeed, this fact follows directly from the commutative square established in Discussion 3.3); regardless, since $d_{n}^{[\varphi]}$ is an injective homomorphism between free left $S$-modules, and $S[\Theta ; \varphi]$ is a flat right $S$-module (cf. Proposition 3.6), one has that $\mathbb{1}_{S[\Theta ; \varphi]} \otimes d_{n}^{[\varphi]}$ is an injective homomorphism between free left $S[\Theta ; \varphi]$-modules. Therefore, $\pi \partial_{n+1}$ is also an injective homomorphism, whence so $\partial_{n+1}$ is. This fact concludes the proof.

Now, we state and prove the first main result of this paper, which is the following:

Theorem 3.9. The $\varphi$-Koszul complex $\mathrm{FK}_{\bullet}\left(x_{1}, \ldots, x_{n}\right)$ provides a free resolution of $S / I_{n}$ in the category of left $S[\Theta ; \varphi]$-modules.

Proof. By Proposition 3.8, it is enough to check, for any $1 \leq l \leq n$, that $H_{l}\left(\mathrm{FK}_{\bullet}\left(x_{1}, \ldots, x_{n}\right)\right)=0$.

So, fix $1 \leq l \leq n$. Our goal is to show that $\operatorname{ker}\left(\partial_{l}\right) \subseteq \operatorname{Im}\left(\partial_{l+1}\right)$; in other words, we have to prove that the chain complex $\mathrm{FK}_{l+1} \stackrel{\partial_{l+1}}{\longrightarrow} \mathrm{FK}_{l} \stackrel{\partial_{l}}{\longrightarrow} \mathrm{FK}_{l-1}$ is midterm exact. First of all, remember that $\mathrm{FK}_{l}=K_{l}^{\prime} \oplus K_{l}^{\prime \prime}$, where

$$
\begin{aligned}
& K_{l}^{\prime}:=\bigoplus_{1 \leq i_{1}<\ldots<i_{l} \leq n} S[\Theta ; \varphi]\left(\mathbf{e}_{i_{1}} \wedge \ldots \wedge \mathbf{e}_{i_{l}}\right), \text { and } \\
& K_{l}^{\prime \prime}:=\bigoplus_{1 \leq j_{1}<\ldots<j_{l-1} \leq n} S[\Theta ; \varphi]\left(\mathbf{e}_{j_{1}} \wedge \ldots \wedge \mathbf{e}_{j_{l-1}} \wedge u\right) .
\end{aligned}
$$

Furthermore, Discussion 3.3 implies that the chain complexes

$$
K_{\bullet}^{\prime}: 0 \longrightarrow K_{n}^{\prime} \stackrel{d_{n}^{\prime}}{\longrightarrow} K_{n-1}^{\prime} \longrightarrow \ldots \longrightarrow K_{2}^{\prime} \stackrel{d_{2}^{\prime}}{\longrightarrow} K_{1}^{\prime} \stackrel{d_{1}^{\prime}}{\longrightarrow} K_{0}^{\prime} \longrightarrow 0
$$

and

$$
\left(K_{\bullet}^{\prime}\right)^{[\varphi]}: 0 \longrightarrow K_{n}^{\prime} \stackrel{\left(d_{n}^{\prime}\right)^{[\varphi]}}{\longrightarrow} K_{n-1}^{\prime} \longrightarrow \ldots \longrightarrow K_{2}^{\prime} \stackrel{\left(d_{2}^{\prime}\right)^{[\varphi]}}{\longrightarrow} K_{1}^{\prime} \stackrel{\left(d_{1}^{\prime}\right)^{[\varphi]}}{\longrightarrow} K_{0}^{\prime} \longrightarrow 0
$$

are respectively canonically isomorphic to $S[\Theta ; \varphi] \otimes_{S} K_{\bullet}$ and $S[\Theta ; \varphi] \otimes_{S} K_{\bullet}^{[\varphi]}$; in particular, since $S[\Theta ; \varphi]$ is a flat right $S$-module (cf. Proposition 3.6), $K_{\bullet}^{\prime}$ and $\left(K_{\bullet}^{\prime}\right)^{[\varphi]}$ are both acyclic chain complexes in the category of left $S[\Theta ; \varphi]$-modules. On the other hand, we also have to keep in mind that $d_{l}^{\prime}$ and $\left(d_{l}^{\prime}\right)^{[\varphi]}$ are respectively represented by right multiplication by matrix $M_{l}$ and $M_{l}^{[\varphi]}$ (cf. Proposition 3.8 and its corresponding notation).

Now, let $P \in \operatorname{ker}\left(\partial_{l}\right) \subseteq \mathrm{FK}_{l}$. Since $\mathrm{FK}_{l}=K_{l}^{\prime} \oplus K_{l}^{\prime \prime}$, we may write $P=\left(P^{\prime} P^{\prime \prime}\right)$ for certain $P^{\prime} \in K_{l}^{\prime}$ and $P^{\prime \prime} \in K_{l}^{\prime \prime}$; in this way, as $P \in \operatorname{ker}\left(\partial_{l}\right)$ it follows that

$$
\left(\begin{array}{ll}
\mathbf{0} & \mathbf{0}
\end{array}\right)=\left(\begin{array}{ll}
P^{\prime} & P^{\prime \prime}
\end{array}\right)\left(\begin{array}{c|c}
M_{l} & \mathbf{0} \\
\hline(-1)^{l-1} D_{l-1} & M_{l-1}^{[\varphi]}
\end{array}\right)=\left(\begin{array}{cc}
P^{\prime} M_{l}+P^{\prime \prime}(-1)^{l-1} D_{l-1} & P^{\prime \prime} M_{l-1}^{[\varphi]}
\end{array}\right),
$$

which leads to the following system of equations:

$$
P^{\prime} M_{l}+P^{\prime \prime}(-1)^{l-1} D_{l-1}=\mathbf{0}, P^{\prime \prime} M_{l-1}^{[\varphi]}=\mathbf{0} .
$$

In particular, since $P^{\prime \prime} M_{l-1}^{[\varphi]}=\mathbf{0}$ one has that $P^{\prime \prime} \in \operatorname{ker}\left(\left(d_{l-1}^{\prime}\right)^{[\varphi]}\right)=\operatorname{Im}\left(\left(d_{l}^{\prime}\right)^{[\varphi]}\right)$; therefore, there is $Q^{\prime \prime} \in K_{l}^{\prime \prime}$ such that $Q^{\prime \prime} M_{l}^{[\varphi]}=P^{\prime \prime}$. Using this fact, it follows that

$$
P^{\prime} M_{l}+Q^{\prime \prime}(-1)^{l-1} M_{l}^{[\varphi]} D_{l-1}=0 .
$$


Regardless, since $M_{l}^{[\varphi]} D_{l-1}=D_{l} M_{l}$ (this has to be proved), we have

$$
P^{\prime} M_{l}+Q^{\prime \prime}(-1)^{l-1} D_{l} M_{l}=0,
$$

which is equivalent to say that $\left(P^{\prime}+Q^{\prime \prime}(-1)^{l-1} D_{l}\right) M_{l}=0$. In this way, one has that

$$
P^{\prime}+Q^{\prime \prime}(-1)^{l-1} D_{l} \in \operatorname{ker}\left(d_{l}^{\prime}\right)=\operatorname{Im}\left(d_{l+1}^{\prime}\right)
$$

and therefore there exists $Q^{\prime} \in K_{l+1}^{\prime}$ such that $Q^{\prime} M_{l+1}=P^{\prime}+Q^{\prime \prime}(-1)^{l-1} D_{l}$.

Summing up, setting $Q:=\left(Q^{\prime} Q^{\prime \prime}\right) \in \mathrm{FK}_{l+1}$, it follows that

$$
\begin{aligned}
\left(\begin{array}{ll}
Q^{\prime} & Q^{\prime \prime}
\end{array}\right)\left(\begin{array}{c|c}
M_{l+1} & \mathbf{0} \\
\hline(-1)^{l} D_{l} & M_{l}^{[\varphi]}
\end{array}\right) & =\left(\begin{array}{ll}
Q^{\prime} M_{l+1}+Q^{\prime \prime}(-1)^{l} D_{l} & Q^{\prime \prime} M_{l}^{[\varphi]}
\end{array}\right) \\
& =\left(\begin{array}{ll}
P^{\prime}+Q^{\prime \prime}(-1)^{l-1} D_{l}+Q^{\prime \prime}(-1)^{l} D_{l} & P^{\prime \prime}
\end{array}\right)=\left(\begin{array}{ll}
P^{\prime} & P^{\prime \prime}
\end{array}\right)
\end{aligned}
$$

and therefore we can conclude that $P \in \operatorname{Im}\left(\partial_{l+1}\right)$, which is exactly what we wanted to show.

The only thing we need to justify is that $M_{l}^{[\varphi]} D_{l-1}=D_{l} M_{l}$; indeed fix $0 \leq l \leq n$. Proposition 3.4 implies that $\partial_{l} \partial_{l+1}=0$. Then, according to Remark 3.5, this equality corresponds to

$$
\left(\begin{array}{c|c}
M_{l+1} & \mathbf{0} \\
\hline(-1)^{l} D_{l} & M_{l}^{[\varphi]}
\end{array}\right)\left(\begin{array}{c|c}
M_{l} & \mathbf{0} \\
\hline(-1)^{l-1} D_{l-1} & M_{l-1}^{[\varphi]}
\end{array}\right)=\mathbf{0} .
$$

In particular, we must have $(-1)^{l} D_{l} M_{l}+(-1)^{l-1} M_{l}^{[\varphi]} D_{l-1}=\mathbf{0}$, which is equivalent to say that

$$
(-1)^{l}\left(D_{l} M_{l}-M_{l}^{[\varphi]} D_{l-1}\right)=\mathbf{0} .
$$

Whence $M_{l}^{[\varphi]} D_{l-1}=D_{l} M_{l}$, as claimed; the proof is therefore completed.

The reader will easily note that, during the proof, we have obtained the result below; we specially thank Rishi Vyas to single out this fact to us.

Proposition 3.10. There is a short exact sequence of chain complexes

$0 \rightarrow S[\Theta ; \varphi] \otimes_{S} K_{\bullet}\left(S ; x_{1}, \ldots, x_{n}\right) \rightarrow \mathrm{FK}_{\bullet}\left(x_{1}, \ldots, x_{n}\right) \rightarrow\left(S[\Theta ; \varphi] \otimes_{S} K_{\bullet}\left(S ; \varphi\left(x_{1}\right), \ldots, \varphi\left(x_{n}\right)\right)\right)[1] \rightarrow 0$ in the category of left $S[\Theta ; \varphi]$-modules.

3.1. The $\varphi$-Koszul chain complex in full generality. Our next aim is to define the $\varphi$-Koszul chain complex over a more general setting, and explore some specific situations on which we can ensure that defines a finite free resolution. Let $A$ be a commutative Noetherian ring containing a commutative subring $\mathbb{K}$, and $y_{1}, \ldots, y_{n}$ denote arbitrary elements of $A$; in addition, assume that we fix a flat endomorphism of $\mathbb{K}$-algebras $S:=\mathbb{K}\left[x_{1}, \ldots, x_{n}\right] \stackrel{\varphi}{\longrightarrow} S$ satisfying $\varphi\left(x_{i}\right) \in\left\langle x_{i}\right\rangle$ for any $1 \leq i \leq n$. In this section, we regard $A$ as an $S$-algebra under the natural homomorphism $S \stackrel{\psi}{\longrightarrow} A$ of $\mathbb{K}$-algebras which sends each $x_{i}$ to $y_{i}$. Finally, we suppose that there exists a $\mathbb{K}$-algebra homomorphism $A \stackrel{\Phi}{\longrightarrow} A$ making the square

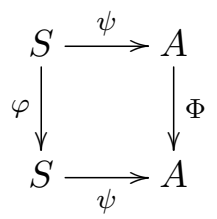

commutative.

Definition 3.11. We define the $\varphi$-Koszul chain complex of $A$ with respect to $y_{1}, \ldots, y_{n}$ as the chain complex FK• $\left(y_{1}, \ldots, y_{n} ; A\right):=A \otimes_{S} \mathrm{FK}_{\bullet}\left(x_{1}, \ldots, x_{n}\right)$. 
Under slightly different assumptions, we want to show that $\mathrm{FK}_{\bullet}\left(y_{1}, \ldots, y_{n} ; A\right)$ still defines a finite free resolution; with this purpose in mind, first of all we review the following notion, which was introduced independently in [Bou07, Definition 2 of page 157] (using a different terminology) and by T. Kabele in [Kab71, Definition 1].

Definition 3.12. Let $R$ be a commutative ring, let $n \in \mathbb{N}$, and let $f_{1}, \ldots, f_{n}$ be a sequence of elements in $R$. It is said that $f_{1}, \ldots, f_{n}$ is a Koszul regular sequence provided the Koszul chain complex $K_{\bullet}\left(f_{1}, \ldots, f_{n} ; R\right)$ provides a free resolution of $R / I_{n}$, where $I_{n}=\left\langle f_{1}, \ldots, f_{n}\right\rangle$.

Next statement may be regarded as a generalization of Theorem 3.9; this is the main result of this section.

Theorem 3.13. Preserving the assumptions and notations established at the beginning of Subsection 3.1, we further suppose that $\Phi$ is flat and $y_{1}, \ldots, y_{n}$ is an A-Koszul regular sequence. Then, FK. $\left(y_{1}, \ldots, y_{n} ; A\right)$ defines a finite free resolution of $A / I_{n}$ in the category of left $A[\Theta ; \Phi]$-modules, where $I_{n}=\left\langle y_{1}, \ldots, y_{n}\right\rangle$.

Proof. The proof of this result is, mutatis mutandis, the same as the one of Theorem 3.9 replacing $S$ by $A$ and $x_{1}, \ldots, x_{n}$ by $y_{1}, \ldots, y_{n}$. Indeed, a simple inspection of the proof of Theorem 3.9 reveals that we only used there the flatness of $\Phi$ and the fact that the Koszul chain complex $K_{\bullet}\left(x_{1}, \ldots, x_{n}\right)$ defines a finite free resolution of $\mathbb{K}$; the proof is therefore completed.

Remark 3.14. The global homological dimension of right skew polynomial rings was studied by K. L. Fields in [Fie69, Fie70]. An upper bound for this global dimension is $n+1$ when $\varphi$ is injective and it is exactly $n+1$ in the case that $\varphi$ is an automorphism. Passing to the opposite ring (see Remark 2.2) we would get analogous results for left skew polynomial rings. Notice that the length of the $\varphi$-Koszul complex FK• $\left(y_{1}, \ldots, y_{n} ; A\right)$ is $n+1$.

3.2. The case of the Frobenius homomorphism. For the convenience of the reader, we will specialize the construction of the $\varphi$-Koszul complex to the case where $A$ is a commutative Noetherian regular ring of positive characteristic $p>0$, and $\varphi=F^{e}$ is an $e$-th iteration of the Frobenius morphism. In this case, we will denote this complex simply as Frobenius-Koszul complex. Namely we have:

$$
\mathrm{FK} \bullet\left(x_{1}, \ldots, x_{n}\right):=0 \longrightarrow \mathrm{FK}_{n+1} \stackrel{\partial_{n+1}}{\longrightarrow} \mathrm{FK}_{n} \stackrel{\partial_{n}}{\longrightarrow} \ldots \stackrel{\partial_{1}}{\longrightarrow} \mathrm{FK}_{0} \longrightarrow 0
$$

where, for each $0 \leq l \leq n+1$,

$$
\mathrm{FK}_{l}:=\bigoplus_{1 \leq i_{1}<\ldots<i_{l} \leq n} A\left[\Theta ; F^{e}\right]\left(\mathbf{e}_{i_{1}} \wedge \ldots \wedge \mathbf{e}_{i_{l}}\right) \oplus \bigoplus_{1 \leq j_{1}<\ldots<j_{l-1} \leq n} A\left[\Theta ; F^{e}\right]\left(\mathbf{e}_{j_{1}} \wedge \ldots \wedge \mathbf{e}_{j_{l-1}} \wedge u\right),
$$

and the differentials $\mathrm{FK}_{l} \stackrel{\partial_{l}}{\longrightarrow} \mathrm{FK}_{l-1}$ are given by:

. For $1 \leq i_{1}<\ldots<i_{l} \leq n$, set

$$
\partial_{l}\left(\mathbf{e}_{i_{1}} \wedge \ldots \wedge \mathbf{e}_{i_{l}}\right):=\sum_{r=1}^{l}(-1)^{r-1} x_{i_{r}}\left(\mathbf{e}_{i_{1}} \wedge \ldots \wedge \mathbf{e}_{i_{r-1}} \wedge \mathbf{e}_{i_{r+1}} \wedge \ldots \wedge \mathbf{e}_{i_{l}}\right) .
$$

. For $1 \leq j_{1}<\ldots<j_{l-1} \leq n$, set

$$
\begin{aligned}
& \partial_{l}\left(\mathbf{e}_{j_{1}} \wedge \ldots \wedge \mathbf{e}_{j_{l-1}} \wedge u\right):=(-1)^{l-1}\left(\Theta-\left(x_{j_{1}}^{p^{e}-1} \cdots x_{j_{l-1}}^{p^{e}-1}\right)\right)\left(\mathbf{e}_{j_{1}} \wedge \ldots \wedge \mathbf{e}_{j_{l-1}}\right) \\
& +\sum_{r=1}^{l-1}(-1)^{r-1} x_{j_{r}}^{p^{e}}\left(\mathbf{e}_{j_{1}} \wedge \ldots \wedge \mathbf{e}_{j_{r-1}} \wedge \mathbf{e}_{j_{r+1}} \wedge \ldots \wedge \mathbf{e}_{j_{l-1}} \wedge u\right) .
\end{aligned}
$$

As a direct consequence of Theorem 3.13 we obtain the below: 
Corollary 3.15. Let $A$ be a commutative Noetherian regular ring of prime characteristic $p$, and let $y_{1}, \ldots, y_{n}$ be an $A-$ Koszul regular sequence. Then, $\mathrm{FK}_{\bullet}\left(y_{1}, \ldots, y_{n} ; A\right)$ defines a finite free resolution of $A / I_{n}$ in the category of left $A\left[\Theta ; F^{e}\right]$-modules, where $I_{n}=\left\langle y_{1}, \ldots, y_{n}\right\rangle$.

Acknowledgements. The authors thank Rishi Vyas for fruitful discussions about the material presented here, and the referee for his/her very useful remarks.

\section{REFERENCES}

[ÀMBZ12] J. Àlvarez Montaner, A. F. Boix, and S. Zarzuela. Frobenius and Cartier algebras of Stanley-Reisner rings. J. Algebra, 358:162-177, 2012. 8

[And00] G. W. Anderson. An elementary approach to L-functions mod p. J. Number Theory, 80(2):291-303, 2000. 7

[BB11] M. Blickle and G. Böckle. Cartier modules: finiteness results. J. Reine Angew. Math., 661:85-123, 2011. 1,6

[Bli13] M. Blickle. Test ideals via algebras of $p^{-e}$-linear maps. J. Algebraic Geom., 22(1):49-83, 2013. 1, 5

[Bou07] N. Bourbaki. Éléments de mathématique. Algèbre. Chapitre 10. Algèbre homologique. Springer-Verlag, Berlin, 2007. Reprint of the 1980 original. 14

[BS13a] M. Blickle and K. Schwede. $p^{-1}$-linear maps in algebra and geometry. In Commutative algebra, pages 123-205. Springer, New York, 2013. 1, 5

[BS13b] M. P. Brodmann and R. Y. Sharp. Local cohomology: an algebraic introduction with geometric applications, volume 136 of Cambridge Studies in Advanced Mathematics. Cambridge University Press, Cambridge, second edition, 2013. 4

[EH08] F. Enescu and M. Hochster. The Frobenius structure of local cohomology. Algebra Number Theory, 2(7):721-754, 2008. 6

[Ene12] F. Enescu. Finite-dimensional vector spaces with Frobenius action. In Progress in commutative algebra 2, pages 101-128. Walter de Gruyter, Berlin, 2012. 1

[EY16] F. Enescu and Y. Yao. The Frobenius complexity of a local ring of prime characteristic. J. Algebra, 459:133-156, 2016. 8

[Fed83] R. Fedder. F-purity and rational singularity. Trans. Amer. Math. Soc., 278(2):461-480, 1983. 8

[Fie69] K. L. Fields. On the global dimension of skew polynomial rings. J. Algebra, 13:1-4, 1969. 14

[Fie70] K. L. Fields. On the global dimension of skew polynomial rings-An addendum. J. Algebra, 14:528-530, 1970. 14

[GW04] K. R. Goodearl and R. B. Warfield, Jr. An introduction to noncommutative Noetherian rings, volume 61 of London Mathematical Society Student Texts. Cambridge University Press, Cambridge, second edition, 2004. 1,3

[HH90] M. Hochster and C. Huneke. Tight closure, invariant theory, and the Briançon-Skoda theorem. J. Amer. Math. Soc., 3(1):31-116, 1990. 1

[Kab71] T. Kabele. Regularity conditions in nonnoetherian rings. Trans. Amer. Math. Soc., 155:363-374, 1971. 14

[KSSZ14] M. Katzman, K. Schwede, A. K. Singh, and W. Zhang. Rings of Frobenius operators. Math. Proc. Cambridge Philos. Soc., 157(1):151-167, 2014. 8

[LS01] G. Lyubeznik and K. E. Smith. On the commutation of the test ideal with localization and completion. Trans. Amer. Math. Soc., 353(8):3149-3180 (electronic), 2001. 1, 5, 6, 8

[Lyu97] G. Lyubeznik. $F$-modules: applications to local cohomology and $D$-modules in characteristic $p>0 . J$. Reine Angew. Math., 491:65-130, 1997. 1

[MR01] J. C. McConnell and J. C. Robson. Noncommutative Noetherian rings, volume 30 of Graduate Studies in Mathematics. American Mathematical Society, Providence, RI, revised edition, 2001. With the cooperation of L. W. Small. 1, 3

[Rot09] J. J. Rotman. An introduction to homological algebra. Universitext. Springer, New York, second edition, 2009. 11

[Sch11] K. Schwede. Test ideals in non-Q-Gorenstein rings. Trans. Amer. Math. Soc., 363(11):5925-5941, 2011. 1, 5

[Sha09] R. Y. Sharp. Graded annihilators and tight closure test ideals. J. Algebra, 322(9):3410-3426, 2009. 1

[SW07] A. K. Singh and U. Walther. Local cohomology and pure morphisms. Illinois J. Math., 51(1):287-298, 2007. 2,9

[SY11] R. Y. Sharp and Y. Yoshino. Right and left modules over the Frobenius skew polynomial ring in the F-finite case. Math. Proc. Cambridge Philos. Soc., 150(3):419-438, 2011. 6

[Yos94] Y. Yoshino. Skew-polynomial rings of Frobenius type and the theory of tight closure. Comm. Algebra, 22(7):2473-2502, 1994. 1, 4, 6, 11 
Departament de Matemàtiques, Universitat Politècnica de Catalunya, Avinguda Diagonal 647, BARCELONA 08028, SPAIN

E-mail address: Josep.Alvarez@upc.edu

Department of Mathematics, Ben-Gurion University of the Negev, P.O.B. 653 Beer-Sheva 84105, ISRAEL.

E-mail address: fernanal@post.bgu.ac.il

Departament d’Àlgebra i Geometria, Universitat de Barcelona, Gran Via de les Corts Catalanes 585, BARCELONA 08007, SPAIN

E-mail address: szarzuela@ub.edu 